\title{
A "photo" album you have not seen; fisheries and oil extraction in the Gulf of Mexico
}

\author{
Magdalena Hernández Chávez ${ }^{1}$, Dora Elia Ramos Muñoz ${ }^{1}$, Lauren Nelson² ${ }^{2}$ Ernesto \\ Llamas $^{2}$, and Alejandro Espinoza Tenorio ${ }^{1}$ \\ ${ }^{1}$ Affiliation not available \\ ${ }^{2}$ Journal of Sketching Science
}

June 15,2020

We all have an album with old photos, a collection of newer digital images and in the future surely we will have many others in more innovative formats. When comparing old photos with recent ones, we can observe how people, society and the environment change over time. Something similar happens with our experiences. We have old images of crucial moments in our history that we store in our album of memories, and we dream of how we want the future to be. From here on, let's imagine that taking a photo at a certain moment in time is similar to collecting information of society and environment for a baseline study.

Years ago, we began to be distant spectators of the relationship between two critical economic activities: fisheries and oil extraction. These two activities take place in the Gulf of Mexico, which includes the coasts of the state of Tabasco. The interaction between both activities is certainly not a voluntary one, but somewhat circumstantial. This fortuitous relationship between fisheries and oil extraction was originated due to the natural wealth specifically concentrated in the Gulf of Mexico.

Through the interaction between these two activities, certain concerns have arisen as to how we can maintain the sustainability of oil extraction and fisheries. To observe the evolution and interaction of such economic activities, we interviewed fishers to obtain valuable historical information stored in their memories and experiences. All fishers recognize that one of the main protagonists implicated in oil extraction has always been the Mexican government, under the name of Petróleos Mexicanos (PEMEX). However, law reforms in 2013 allowed private companies to open new areas of exploitation in the Gulf of Mexico (EspinozaTenorio, 2019). The recollected images or historical information gave us an insight as to how different actors modified fisheries and oil extraction. On the other hand, the most recent information collected were similar to "panoramic digital photos" showing all actors that coexist on the coasts of the Gulf of Mexico. Historical and current information were like old and new "photos" taken with the highest possible resolution to observe in detail how both activities modify the landscape. From this information or "photos" we obtained, we could extract relevant data to improve the sustainability of both coastal activities.

\section{Who took the "photos"?}

Twenty-five academics from five different research institutions formed a multidisciplinary team that, for two years (2017-2019), took and collected like "photos" alongside the local population. We selected older and more recent "photos" which showed a wide vision of the richness of marine nature, and relevant historical elements. The most relevant "photos" showed the complex scenario where fisheries and oil extraction were competing and affecting the coastal communities.

\section{How did people take or recollect the "photos"?}

Project participants used different approaches like photographers use different equipment like cameras, 


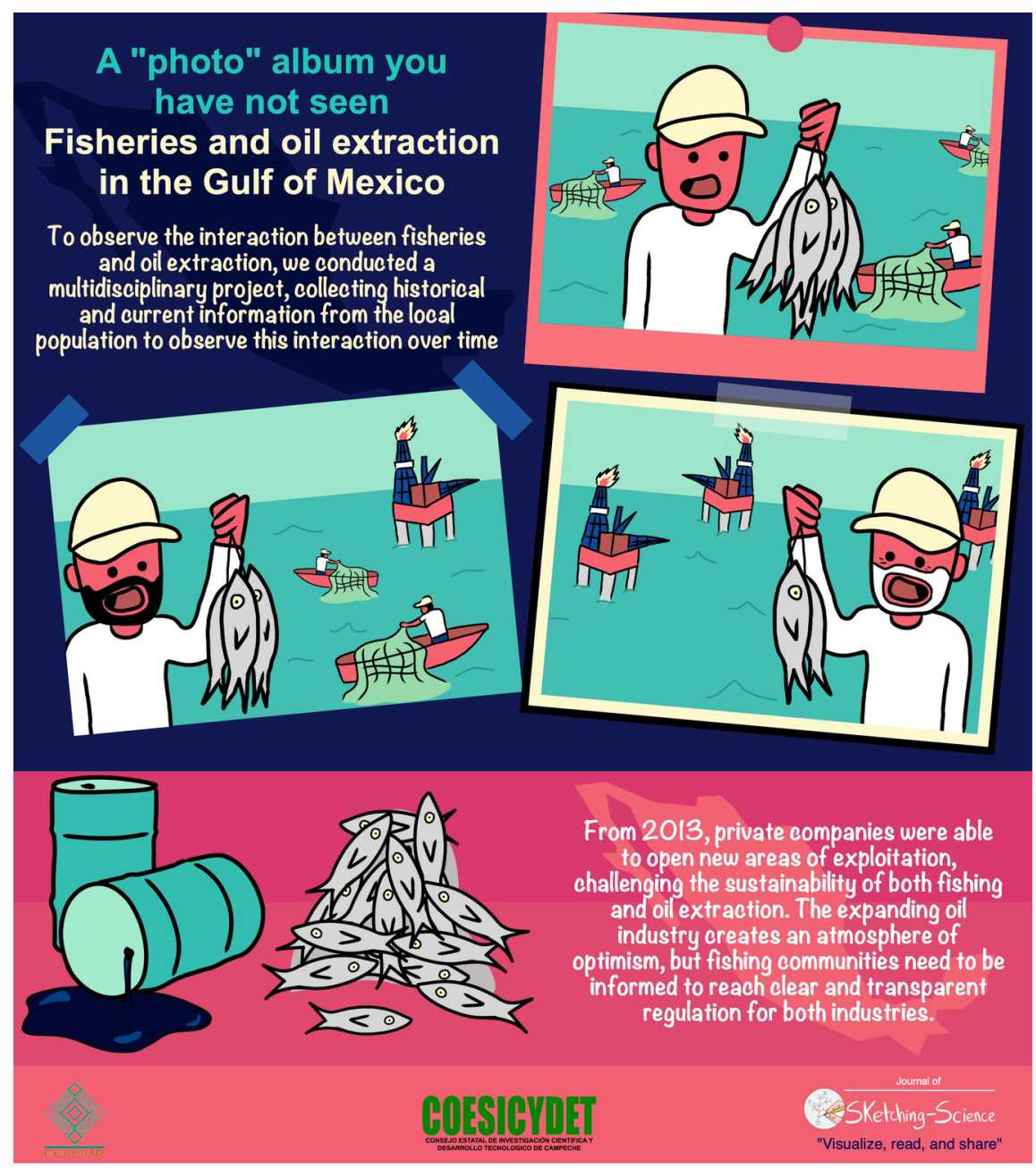

tripods, and lenses to capture how they perceive fisheries and oil extraction. Some "photos" however, were obtained from historical collections, academic research or government institutions.

\section{What did we find in the "photos"?}

In general, this information or "photos" captured the relationship between fishers and oil workers over the years. Such a relationship has gone from formal to informal, and from encounters to disagreements. We have identified more than 50 social visible actors, such as PEMEX, fisheries cooperatives, authorities, universities, civil society organisations, and women and children [who are affected]. In this governance network some "rules of the game" have been created which allow both activities to coexist in an unequal and changing game (Salazar-de $a_{C}$ ruzetal., 2020).

We found that fishers receive financial compensation for the impacts of oil extraction, but feel that their vocation is not taken into account and are being dispossessed of their produce (Ramos-Muñoz 2019). Fishers also feel that they work in a context of insecurity and distrust, where they have been gradually excluded and abandoned.

We were also able to identify which species of fish had more economic relevance. Moreover, we found that there are about 32 fisheries in the Tabasco and Campeche coasts, where mussel farming and red snapper fishing have potential to meet sustainable goals shortly, but more research is needed. Importantly, we also analyzed the role of women in fisheries, an activity traditionally dominated by men. 
Another significant finding is that fishing provides food to fishers homes, representing $30 \%$ of their total income (Palacios-Hernández, 2019).

Finally, in order to improve the sustainability of both activities, we must pay special attention to some "photos" captured in the communities: 1) the majority of young people and women in these coastal communities show willingness to expand their productive activities; an example is aquaculture; 2) the revival of the oil industry creates an atmosphere of optimism, but communities would like to be better informed; 3) it is urgent to build links with the communities to improve communication and reach agreements with a capacity for consensus, under clear and transparent rules.

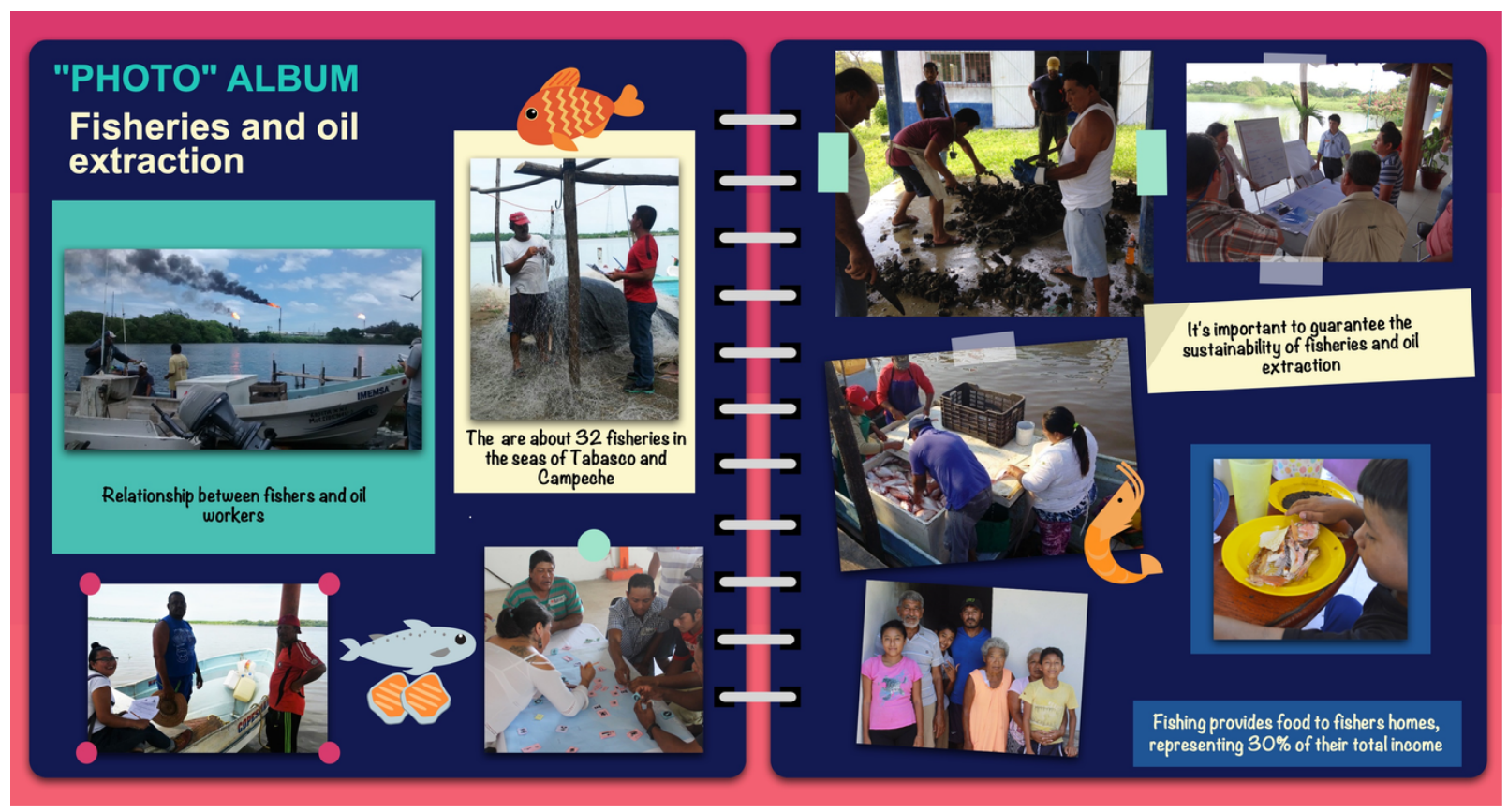

Who do we give the "photos" to?

At the end of the project, the "photos" were distributed amongst the participants so they could recognize themselves and their neighbors. The "photos" are also for those who could not participate, but wanted to meet us; know who we are, what we wanted and what we aspired to do. Therefore, together we can decide what we want to achieve and, in the future, check if what we decided upon took us where we wanted to go. More "photos" and information in www.pescaypetroleo.info

\section{Here is a video about fisheries and oil extraction}

If you want more information about fisheries and oil extraction in the Gulf of Mexico, take a look to the next video (Spanish, with English subtitles).

Rich media available at https://www .youtube.com/watch?v=EnEvRCxZIQU\&feature=youtu . be 


\section{Un álbum de "fotos" que no has visto; la pesca y la extracción de petróleo en el Golfo de México}

Todos tenemos un álbum con fotos antiguas, una colección de imágenes digitales más recientes y en el futuro, seguramente, tendremos otras tantas en formatos más innovadores. Cuando comparamos fotos antiguas con recientes podemos observar cómo la gente, la sociedad y el ambiente cambian a través del tiempo. Algo similar ocurre con nuestras experiencias. Tenemos imágenes antiguas de momentos cruciales en nuestra historia que almacenamos en nuestro álbum de recuerdos, y soñamos cómo queremos que sea el futuro. En adelante, imaginemos que tomar una foto en un momento determinado es similar a recopilar información de la sociedad y el medio ambiente para un estudio base.

Años atrás, comenzamos a ser espectadores distantes de la relación que sostenían dos críticas actividades económicas: la pesca y la extracción de petróleo. Estas dos actividades tienen lugar en el Golfo de México, que incluye las costas del estado de Tabasco. La interacción entre ambas actividades no es ciertamente voluntaria, sino más bien circunstancial. Esta fortuita relación entre la pesca y la extracción de petróleo se originó debido a las riquezas naturales concentradas específicamente en el Golfo de México.

A través de la interacción entre estas dos actividades han surgido ciertas preocupaciones sobre cómo podemos mantener la sostenibilidad de la extracción de petróleo y la pesca. Para observar la evolución de la interacción de semejantes actividades económicas, entrevistamos a personas dedicadas a la pesca para obtener valiosa información histórica almacenada en sus recuerdos y experiencias. Todas ellas reconocen que uno de los principales protagonistas implicados en la extracción del petróleo ha sido el gobierno mexicano, bajo el nombre de Petróleos Mexicanos (PEMEX). Sin embargo, las reformas a las leyes en 2013 permitieron a empresas privadas abrir nuevas áreas de explotación en el Golfo de México (Espinoza-Tenorio, 2019). Las imágenes recolectadas o la información histórica nos dieron una idea de cómo los diferentes actores modificaron la pesca y la extracción de petróleo. Por otro lado, la información más reciente que se recopiló fue similar a "fotos digitales panorámicas" que muestran a todos los actores que coexisten en las costas del Golfo de México. La información histórica y actual fueron como "fotos" antiguas y nuevas tomadas con la mayor resolución posible para observar a detalle cómo ambas actividades modifican el paisaje. A partir de esta información o "fotos" obtenidas, podríamos extraer datos relevantes para mejorar la sostenibilidad de ambas actividades costeras.

\section{¿Quiénes tomaron las" fotos"?}

Veinticinco académicos de cinco diferentes institutos de investigación formaron un equipo multidisciplinario que, durante dos años (2017-2019), tomó y recolectó estas "fotos" junto a la población local. Seleccionamos las "fotos" más antiguas y recientes que mostraran una amplia visión de la riqueza de la naturaleza marina y los elementos históricos relevantes. Las "fotos" más relevantes mostraron el complejo escenario en el que la pesca y la extracción de petróleo estaban compitiendo y afectando a las comunidades costeras.

\section{¿Cómo tomaron o recolectaron las "fotos"?}

Los participantes del proyecto utilizaron diferentes enfoques, así como los fotógrafos usan diferentes equipos como cámaras, tripiés y lentes para capturar cómo perciben la pesca y la extracción de petróleo. Sin embargo, algunas "fotos" se obtuvieron de colecciones históricas, investigaciones académicas o instituciones gubernamentales.

\section{¿Qué encontramos en las "fotos"?}




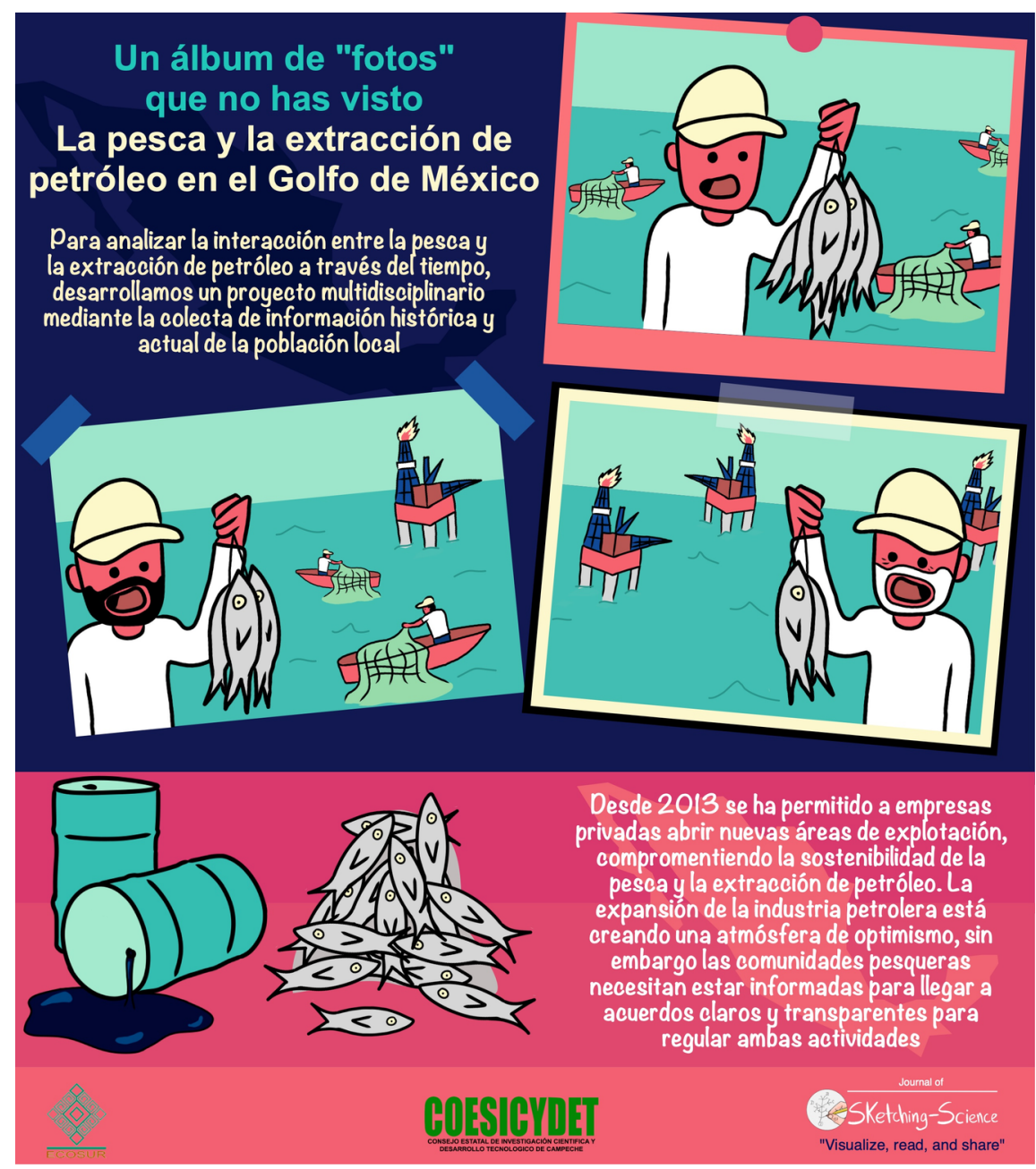

En general, esta información o "fotos" capturaron la relación entre personas dedicadas a la pesca y trabajadores petroleros a lo largo de los años. Tal relación ha ido de lo formal a lo informal y del encuentro al desencuentro. Hemos identificado a más de 50 actores sociales visibles como PEMEX, cooperativas pesqueras, autoridades, universidades, organizaciones de la sociedad civil, y mujeres y niños [que se ven afectados]. En esta red de gobierno se han creado algunas "reglas del juego" que permiten que ambas actividades coexistan en un juego desigual y cambiante (Salazar- $\mathrm{de}_{l} a_{C}$ ruzetal., 2020).

Descubrimos que las personas dedicadas a la pesca reciben una compensación financiera por el impacto que causa la extracción de petróleo, pero sienten que su vocación no se tiene en cuenta y están siendo desposeídos de su actividad productiva (Ramos-Muñoz 2019). Estas personas también sienten que trabajan en un contexto de inseguridad y desconfianza, donde fueron gradualmente excluidos y abandonados.

También pudimos identificar qué especies de peces tenían mayor relevancia económica. Además, descubrimos que hay alrededor de 32 pesquerías en las costas de Tabasco y Campeche, donde la cría de mejillones y la pesca de pargo rojo tienen el potencial para alcanzar metas sostenibles en breve, pero se necesita más investigación al respecto. Es importante destacar que también analizamos el papel de las mujeres en la pesca, una actividad tradicionalmente dominada por los hombres.

Otro hallazgo significativo es que la pesca provee alimento a los hogares de la gente que se dedica a esta actividad, lo que representa el 30\% de sus ingresos totales (Palacios-Hernández, 2019). 
Finalmente, para mejorar la sostenibilidad de ambas actividades, debemos prestar especial atención a algunas "fotos" capturadas en las comunidades: 1) la mayoría de los jóvenes y las mujeres en estas comunidades costeras muestran disposición para expandir sus actividades productivas; un ejemplo es la acuicultura; 2) el renacimiento de la industria petrolera crea una atmósfera de optimismo, pero a las comunidades les gustaría estar mejor informadas; 3) es urgente establecer vínculos con las comunidades para mejorar la comunicación y alcanzar acuerdos con capacidad de consenso, bajo reglas claras y transparentes.

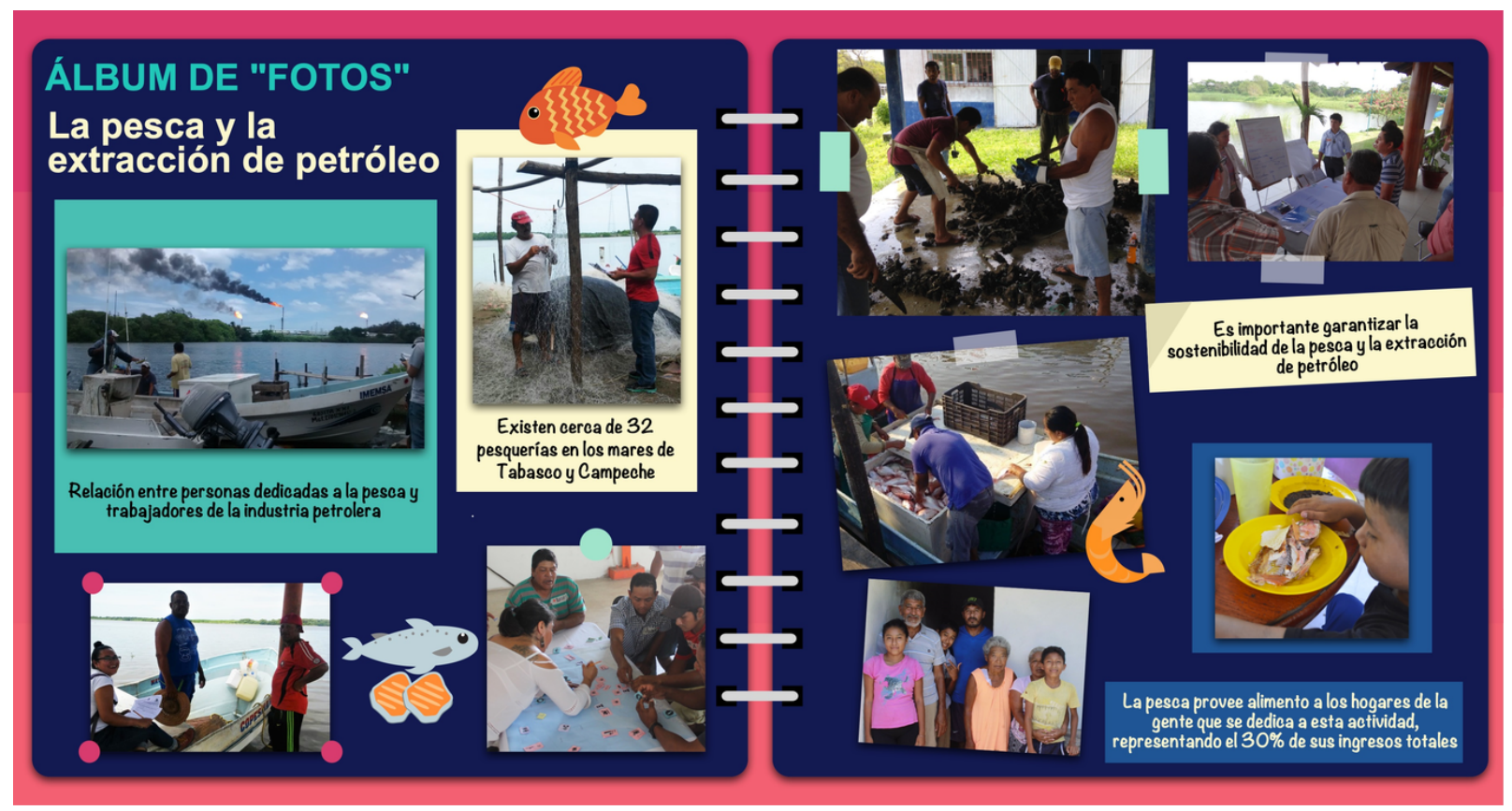

\section{¿A quién le regalamos las "fotos"?}

Al final del proyecto, las "fotos" se distribuyeron entre los participantes para que pudieran reconocerse a sí mismos y a sus vecinos. Las "fotos" también son para aquellos que no pudieron participar, pero quisieron conocernos; saber quiénes somos y qué aspiramos a hacer. Así, juntos podemos decidir lo que queremos lograr y, en el futuro, verificar si lo que decidimos nos llevó a donde queríamos ir. Más "fotos" e información en www.pescaypetroleo.info

Aquí hay un vídeo acerca de la pesca y la extracción de petróleo

Si quieres más información acerca de la pesca y la extracción de petróleo en el Golfo de México, mira el siguiente vídeo (español, con subtítulos en inglés).

Rich media available at https://www $\cdot$ youtube.com/watch?v=EnEvRCxZIQU\&feature=emb_title

\section{Contributions}

Magdalena Hernández Chávez wrote and edited the article. She is an academic technician at El Colegio de la Frontera Sur in Villahermosa, Mexico. She works at the Laboratorio Transdisciplinario para la Sustentabilidad (LaTSu) and is interested in science communication.

Dora Elia Ramos-Muñoz wrote the article. She is a professor and researcher of El Colegio de la Frontera Sur in Villahermosa, Mexico. She studies regional development, extractive processes and explore participatory 
methodologies. (Twitter @doraeliaramos).

Lauren Nelson edited the article. Lauren is a Ph.D. student at Newcastle University (UK), researching computational drug design alongside the Northern Institute for Cancer Research. Lauren also writes a scientific blog, bringing science to the masses. (Twitter (@ashortscientist); Facebook (@ashortscientist); Instagram (@ashortscientist); Blog: ashortscientist.wordpress.com).

Ernesto Llamas edited the article and made the illustrations. He is currently a postdoctoral researcher at CECAD in Cologne, Germany. He obtained his Ph.D. in Biotechnology from Universitat de Barcelona doing his research at the Centre for Research in Agricultural Genomics. Creator, editor, and illustrator of Sketching Science. (Twitter @neto_flames; Instagram @eellamas).

Alejandro Espinoza-Tenorio wrote the article. He is a professor and researcher of El Colegio de la Frontera Sur. He obtained his PhD from the University of Bremen, Germany and his experience has led him to develop and test methodologies on holistic management in coastal areas. (Twitter @espinatenorio).

\section{References}

Espinoza-Tenorio A. 2019. ¿Mancharse las manos de negro? El dilema ético de la investigación en territorios petrolizados. Rev Mex Cienc Polit Soc LXIV:183-210.

Palacios-Hernández JM. 2019. Estimación del servicio ecosistémico de provisión de alimento en la costa de Tabasco. El caso del municipio de Centla, Tabasco (Master's thesis). Instituto Tecnológico Superior de Centla.

Ramos-Muñoz D, Ramos-Reyes R, Zamora-Cornelio LF, la Cruz AH-D, Espinoza-Tenorio A. 2019. Exclusión en el Golfo de México: una visión desde los pescadores sobre la industria petrolera en Tabasco. Cuadernos de Geografía: Revista Colombiana de Geografía 28:357-372. doi:10.15446/rcdg.v28n2.73511

Salazar-de $a_{C}$ ruzC,Zepeda $\quad-\quad$ DominguezJA,Espinoza $\quad-\quad$ TenorioA, Ramos MuñozDE.2020.Governancenetworksinmarinespaceswherefisheriesandoilcoexist: TabascoMéxico. The Extractive Indus 10.1016/j.exis.2020.03.012 